\title{
Tuberculous Peritonitis Mimicking Malignancy during Adalimumab Therapy in a Patient with Rheumatoid Arthritis
}

\author{
Refik Demirtunç ${ }^{1}$, Uğur Özensoy ${ }^{1}$, Kadir Kayataş ${ }^{1}$, Fügen Vardar Aker ${ }^{2}$ \\ ${ }^{1}$ Department of Internal Medicine, Haydarpaşa Numune Training and Research Hospital, İstanbul, Turkey \\ ${ }^{2}$ Department of Pathology, Haydarpaşa Numune Training and Research Hospital, İstanbul, Turkey \\ Email: rdemirtunc@hotmail.com, ozensoy@hotmail.com, kadirkayatas@yahoo.com, fugenaker@yahoo.com
}

Received 16 June 2015; accepted 24 August 2015; published 27 August 2015

Copyright (C) 2015 by authors and Scientific Research Publishing Inc.

This work is licensed under the Creative Commons Attribution International License (CC BY). http://creativecommons.org/licenses/by/4.0/

c) (i) Open Access

\begin{abstract}
We present a case of tuberculous peritonitis in a 46-year-old woman with rheumatoid arthritis treated with adalimumab, and we review the association between anti-tumour necrosis factor therapy and tuberculosis. This case illustrates that tuberculosis can develop with atypical clinic and uncertain laboratory findings and may mimic malignancies during adalimumab therapy.
\end{abstract}

\section{Keywords}

\section{Adalimumab, Rheumatoid Arthritis, Tuberculous Peritonitis}

\section{Introduction}

Anti-tumor necrosis factor (anti-TNF) drugs have been used for more than 10 years to treat inflammatory conditions such as rheumatoid arthritis (RA), psoriatic arthritis, juvenile arthritis, inflammatory bowel diseases, ankylosing spondylitis and psoriasis [1].

Post marketing surveillance has showed that long-term use of anti-TNF agents may increase the risk of developing serious infections, cancers such as lymphoma and skin cancer, lupus-like autoimmune disease, liver disease, demyelinating disorders and hematologic abnormalities [2]. According to reports, tuberculosis (TB) is the most frequent opportunistic, infection during anti-TNF drug therapy [3]. Anti-TNF- $\alpha$ therapy has been shown to be associated with an approximately 14-fold greater incidence of TB reactivation compared to healthy controls [4]. In vitro studies suggest that TNF- $\alpha$ plays an important role in the regulation of granuloma formation, which serves to restrict bacterial growth [5] [6], enhances macrophage activation [7], chemokine production by macrophages [8] and immune cell recruitment during M. tuberculosis infection [9]. Anti-TNF- $\alpha$ monoc- 
lonal antibody administration may subsequently result in the dissolution of intact granulomas, the release of viable mycobacteria, and disease reactivation [10]. This can explain the higher incidence of TB observed in patients receiving anti TNF- $\alpha$ treatment [4]. Highest TB risk appears with infliximab and may reach five to ten times that of untreated patients [11] [12]. Although data regarding adalimumab are not sufficient, etanercept and adalimumab seems to be associated with a slightly increased risk [12]-[15]. TB developing during anti-TNF therapy may be reactivation of latent TB or newly developed infection, and may have localized or disseminated manifestation [16].

Herein, we present a case of tuberculous peritonitis in a patient with rheumatoid arthritis treated with adalimumab.

\section{Case Report}

A 46-year-old female with a long history of RA, on treatment with methotrexate and adalimumab was admitted to the hospital because of ascites, low abdominal pain that had developed in the previous 5 weeks. She had unintentionally lost eight kilograms in last year. TB screening was performed before adalimumab and her purified protein derivative (PPD) test was $16 \mathrm{~mm}$, with a normal chest radiograph and previous BCG vaccination. The patient denied previous history or known exposure to TB. She had been treated with $300 \mathrm{mg} /$ day isoniazid for 9 months for latent TB. Then adalimumab (subcutaneous injection of $40 \mathrm{mg}$ every other week) was started 48 months before admission, with good tolerance and clinical result. She was being treated with methotrexate 15 $\mathrm{mg} /$ week and prednisolone $5 \mathrm{mg} /$ day for 2 year before adalimumab started. Physical examination revealed only ascites and tenderness on deep palpation. Recent PPD test was anergic. Laboratory evaluation showed 6100 white blood cells per microlitre (66\% neutrophil, 19\% lymphocyte, 14\% monocyte), and hemoglobin $12.7 \mathrm{~g} / \mathrm{dL}$. Erythrocyte sedimentation rate was $26 \mathrm{~mm}$. Electrolytes, liver, and renal functions tests, thyroid-stimulating hormone, antinuclear antibodies, hepatitis virus and HIV serology were normal or negative. Rheumatoid factor was negative. CA 125 level was $487.1 \mathrm{IU} / \mathrm{mL}$ (normal < $35 \mathrm{IU} / \mathrm{mL}$ ).

Ascites fluid analysis revealed albumin $2.1 \mathrm{~g} / \mathrm{dL}$ (simultaneous serum albumin level of $2.7 \mathrm{~g} / \mathrm{dL}$ and SAAG was 0.6), LDH $483 \mathrm{U} / \mathrm{L}$ (simultaneous serum LDH level of $216 \mathrm{U} / \mathrm{L}$ ), 2370 white cell per microlitre (63\% lymphocyte), adenosine deaminase level 17U/L (normal $<40 \mathrm{U} / \mathrm{L}$ ). Quantiferon GOLD test yielded indefinite result. Cytological examination of ascites revealed lymphocyte predominance and non-neoplastic cytological findings. Histopathological examination of peritoneal biopsy revealed well-formed granuloma formation with Langhans type multinuclear giant cell and caseating necrosis in peritoneal surface (Figure 1 and Figure 2). TB peritonitis

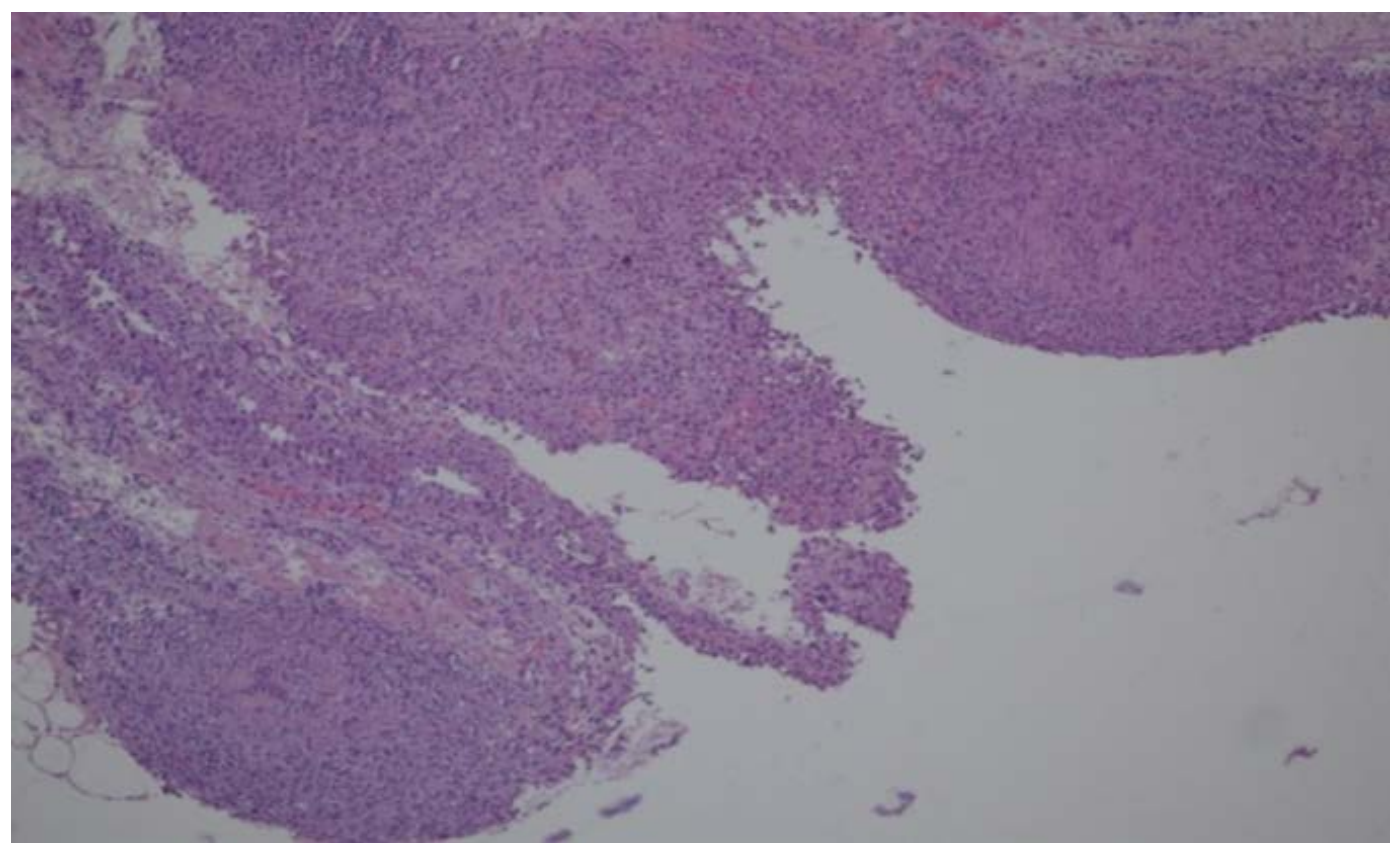

Figure 1. Extensive gralunomatous reactions in peritoneal surface (H \& E ×40). 


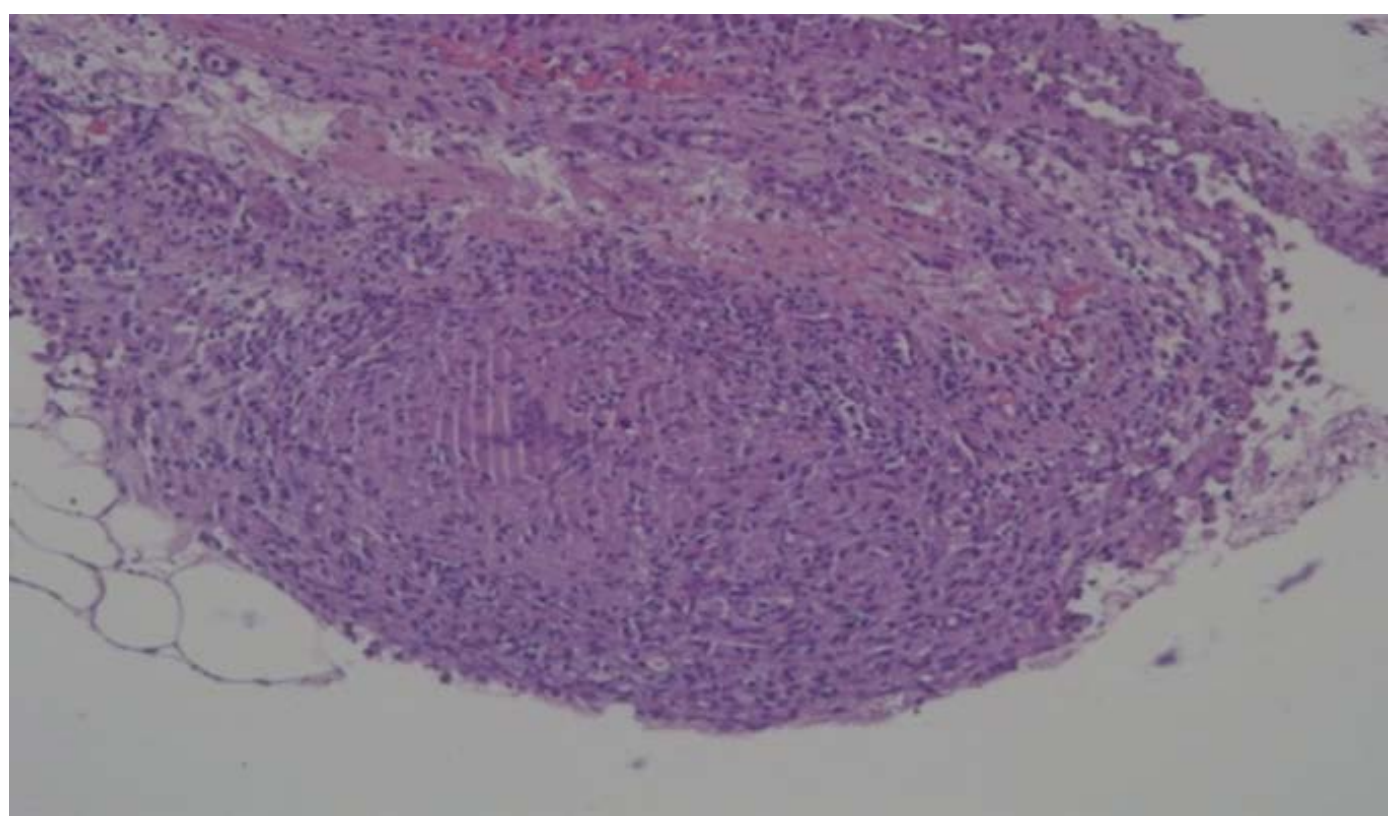

Figure 2. Well formed granuloma formation with Langhanstype cell and caseating necrosis (H \& E ×200).

was thought primarily but mycobacteria were not identified by EZN histochemistry on paraffin tissue section and acid-fast staining in the ascitic fluid. While waiting culture results, with this histopathological findings, adalimumab was discontinued and antituberculous therapy with isoniazid $5 \mathrm{mg} / \mathrm{kg}$, rifampin $10 \mathrm{mg} / \mathrm{kg}$, ethambutol $20 \mathrm{mg} / \mathrm{kg}$ and pyrazinamide $25 \mathrm{mg} / \mathrm{kg}$ was started for first two month. A negative result for ascitic fluid culture was obtained 8 week after the initiation of antituberculous therapy.

Chest radiography and thorax computed tomography were normal. Abdominal CT scan showed widespread retroperitoneal lymphadenopathy, widespread free ascites, millimetric peritoneal nodules, peritoneal thickening and $2.5 \times 3 \mathrm{~cm}$ in diameter lobular contoured nodular density in the right adnexial area suggesting ovarian malignancy. The patient has given her consent for the case report to be published.

\section{Discussion}

TB peritonitis is a quite rare condition. Approximately 70 percent of patients have symptoms such as abdominal pain, fever, and weight loss. for more than four months before the diagnosis is established. The most common features were ascites (93\%), abdominal pain (73\%), and fever (58\%). TB peritonitis comprises $12 \%$ of extrapulmonary TB cases [17]. When we reviewed the literature on PubMed, there are few cases with anti-TNF therapy-associated TB peritonitis and only 6 TB peritonitis cases have been reported after adalimumab treatment. Most of anti-TNF treatment-associated TB cases are activation of latent TB infections and occurs in short time after the initiation of Anti-TNF treatment. Although anti-TNF treatment-associated TB occurs in the early months after infliximab treatment, (12 - 21 weeks), suggesting that reactivation of latent TB [5], the development of TB in etanercept treated patients occurs after the initiation of therapy with average 11.5 months [15], suggesting that newly infected TB. The average appearance time of TB was 16.6 months in adalimumab associated 6 cases (36-18-12-12-11-11 months). Only one of these cases had positive tuberculin skin test before adalimumab treatment and took anti-TB treatment before the adalimumab [17]. Therefore, these 6 case should be thought as newly infected TB. Our patient had $16 \mathrm{~mm}$ PPD test and TB occured 48 months after the initiation of adalimumab treatment, therefore it should be thought as newly developed TB.

In our patient, Mycobacterium could not be shown by acid-fast staining in the ascitic fluid. Examination of an acid fast stained smear of ascitic fluid has a disappointingly low yield. The direct smear of ascitic fluid has only 0 to 6 percent sensitivity for detecting Mycobacteria [18]. Ascitic fluid culture was negative for mycobacterium tuberculosis in our patient. In most series, the frequency of a positive ascites culture is disappointingly less than 20 percent [19]. The utility of cultures is even more questionable when considering the delay of four to six weeks before a result is obtained. The delay can be associated with increased mortality [20]. 
In our patient, peritoneal biopsy histopathological examination revealed extensive gralunomatous reactions with Langhans type multinuclear giant cell and caseating necrosis compatible with TB peritonitis. However, TB bacilli could not be shown by EZN histochemistry on paraffin tissue section. In addition to these, the high incidence of TB in our country led us to strongly think of TB.

After two months of therapy with isoniazid, rifampin, ethambutol and pyrazinamide have been completed, clinic of our patient was improved, ascite and findings considerning malignancy disappeared in the control Abdominal CT. Four more months' antituberculous therapy with isoniazid and rifampin was planned for full recovery.

Although ascitic fluid culture was negative for mycobacterium tuberculosis, the diagnosis of TB was confirmed with respond to treatment and malignancy was excluded.

As a result, in countries such as Turkey which has a high incidence of TB, before the initiation of the anti-TNF- $\alpha$ therapy, latent TB screening must be done appropriately. The patients with latent TB should take adequate dose and duration chemoprophylaxis and compliance to this therapy should be monitored carefully. We suggest performing early diagnostic peritoneal biopsy for patients with unexplained ascites, especially in localities where tuberculosis is prevalent. In case of having strong suspicion of TB, although not certain laboratory findings and typical clinic to confirm the TB diagnosis, initiation of anti-TB therapy is seen as a rational approach, because of possible high morbidity and mortality in case of late initiation of treatment. It should not be forgotten that intra-abdominal TB developed after anti-TNF therapy may mimic malignancies.

\section{References}

[1] Toussirot, E., Streit, G. and Wendling, D. (2007) Infectious Complications with Anti-TNFalpha Therapy in Rheumatic Diseases: A Review. Recent Patents on Inflammation \& Allergy Drug Discovery, 1, 39-47. http://dx.doi.org/10.2174/187221307779815039

[2] Olsen, N.J., Michael Stein, C. and Ch.B., M.B. (2004) New Drugs for Rheumatoid Arthritis. New England Journal of Medicine, 350, 2167-2179. http://dx.doi.org/10.1056/NEJMra032906

[3] Antolín, J., Azahara, M., Hernández, C., Blanco, M., Mao, L. and Cigüenza, R. (2008) Scand Tuberculous Peritonitis after Treatment with Adalimumab. Journal of Infectious Diseases, 40, 677-678.

[4] Yoo, I.K., Choung, R.S., Hyun, J.J., Kim, S.Y., Jung, S.W., Koo, J.S., et al. (2014) Incidences of Serious Infections and Tuberculosis among Patients. Yonsei Medical Journal, 55, 442-448. http://dx.doi.org/10.3349/ymj.2014.55.2.442

[5] Saunders, B.M., Briscoe, H. and Britton, W.J. (2004) T Cell-Derived Tumour Necrosis Factor Is Essential, but Not Sufficient, for Protection against Mycobacterium tuberculosis Infection. Clinical \& Experimental Immunology, 137, 279-287. http://dx.doi.org/10.1111/j.1365-2249.2004.02518.x

[6] Flynn, J.L., Goldstein, M.M., Chan, J., Triebold, K.J., Pfeffer, K., Lowenstein, C.J., et al. (1995) Tumor Necrosis Factor-Alpha Is Required in the Protective Immune Response against Mycobacterium tuberculosis in Mice. Immunity, 2, 561-572. http://dx.doi.org/10.1016/1074-7613(95)90001-2

[7] Harris, J., Hope, J.C. and Keane, J. (2008) Tumor Necrosis Factor Blockers Influence Macrophage Responses to Mycobacterium tuberculosis. Journal of Infectious Diseases, 198, 1842-1850. http://dx.doi.org/10.1086/593174

[8] Algood, H.M., Lin, P.L., Yankura, D., Jones, A., Chan, J. and Flynn, J.L. (2004) TNF Influences Chemokine Expression of Macrophages in Vitro and That of CD11b+ Cells in Vivo during Mycobacterium tuberculosis Infection. Journal of Immunology, 172, 6846-6857. http://dx.doi.org/10.4049/jimmunol.172.11.6846

[9] Zhou, Z., Connell, M.C. and MacEwan, D.J. (2007) TNFR1-Induced NF-KappaB, but Not ERK, p38MAPK or JNK Activation, Mediates TNF-Induced ICAM-1 and VCAM-1 Expression on Endothelial Cells. Cell Signal, 19, 12381248. http://dx.doi.org/10.1016/j.cellsig.2006.12.013

[10] Keane, J. and Bresnihan, B. (2008) Tuberculosis Reactivation during Immune Suppressive Therapy in Rheumatic Diseases: Diagnostic and Therapeutic Strategies. Current Opinion in Rheumatology, 20, 443-449. http://dx.doi.org/10.1097/BOR.0b013e3283025ec2

[11] Bieber, J. and Kavanaugh, A. (2004) Consideration of the Risk and Treatment of Tuberculosis in Patients Who Have Rheumatoid Arthritis and Receive Biologic Treatments. Rheumatic Disease Clinics of North America, 30, 257-270. http://dx.doi.org/10.1016/j.rdc.2004.01.003

[12] Rychly, D.J. and Dipiro, J.T. (2005) Infections Associated with Tumor Necrosis Factor-Alpha Antagonists. Pharmacotherapy, 25, 1181-1192. http://dx.doi.org/10.1592/phco.2005.25.9.1181

[13] Gutiérrez-Macías, A., Lizarralde-Palacios, E., Martínez-Odriozola, P. and Miguel-de la Villa, F. (2007) Tuberculous Peritonitis in a Patient with Rheumatoid Arthritis Treated with Adalimumab. Clinical Rheumatology, 26, 452-453. 
http://dx.doi.org/10.1007/s10067-005-0164-3

[14] Scheinfeld, N. (2005) Adalimumab: A Review of Side Effects. Expert Opinion on Drug Safety, 4, 637-641. http://dx.doi.org/10.1517/14740338.4.4.637

[15] Mohan, A.K., Cote, T.R., Block, J.A., Manadan, A.M., Siegel, J.N. and Braun, M.M. (2004) Tuberculosis Following the Use of Etanercept, a Tumor Necrosis Factor Inhibitor. Clinical Infectious Diseases, 39, 295-299. http://dx.doi.org/10.1086/421494

[16] Yoo, W.H. (2012) Multiple Organ Tuberculosis of Lung, Pleura, and Peritoneum in Ankylosing Spondylitis during Adalimumab Therapy. Rheumatology International, 32, 787-790. http://dx.doi.org/10.1007/s00296-009-1357-x

[17] Dascalu, C., Valceanu, A., Brennessel, D. and Bandagi, S. (2013) New Therapies, Increased Risk for Old InfectionsAbdominal Tuberculosis Mimicking Colon Cancer during Adalimumab Treatment for Rheumatoid Arthritis. Journal of Clinical Rheumatology, 19, 297-299. http://dx.doi.org/10.1097/RHU.0b013e31828c3d73

[18] Chow, K.M., Chow, V.C. and Szeto, C.C. (2003) Indication for Peritoneal Biopsy in Tuberculous Peritonitis. The American Journal of Surgery, 185, 567-573. http://dx.doi.org/10.1016/S0002-9610(03)00079-5

[19] Marshall, J.B. (1993) Tuberculosis of the Gastrointestinal Tract and Peritoneum. American Journal of Gastroenterology, 88, 989-999.

[20] Chow, K.M., Chow, V.C., Hung, L.C., Wong, S.M. and Szeto, C.C. (2002) Tuberculous Peritonitis-Associated Mortality Is High among Patients Waiting for the Results of Mycobacterial Cultures of Ascitic Fluid Samples. Clinical Infectious Diseases, 35, 409-413. http://dx.doi.org/10.1086/341898 